\title{
Evaluation of rumen-protected lysine supplementation to lactating dairy cows consuming increasing amounts of distillers dried grains with solubles
}

\author{
H. A. Paz, ${ }^{*}$ M. J. de Veth, $†$ R. S. Ordway, $†$ and P. J. Kononoff ${ }^{* 1}$ \\ ${ }^{*}$ Department of Animal Science, University of Nebraska-Lincoln, Lincoln 68583 \\ †Balchem Corporation, New Hampton, NY 10958
}

\begin{abstract}
Twenty multiparous Holstein cows were used in four $5 \times 5$ Latin squares to determine the effects of feeding increasing amounts of distillers dried grains with solubles (DDGS) in diets with or without the supplementation $(60 \mathrm{~g} / \mathrm{d})$ of a rumen-protected Lys (RPL) product (AminoShure-L, 38\% L-Lys; Balchem Encapsulates, New Hampton, NY) on milk yield and composition and plasma concentration of AA. Dietary treatments were (1) control (CON; no DDGS), (2) $10 \%$ DDGS (10DG), (3) 20\% DDGS (20DG), (4) 10\% DDGS plus RPL (10DGRPL), and (5) 20\% DDGS plus RPL (20DGRPL). Diets were formulated using the CornellPenn-Miner Dairy model (CPM v3.0; http://cahpwww. vet.upenn.edu/node/77) to provide a predicted decreasing supply of Lys $(117,99$, and $91 \%$ of requirements) for the CON, 10DG, and 20DG diets, respectively. Addition of RPL to the 10DG and 20DG diets (unsupplemented diets) resulted in 2 additional treatments, 10DGRPL and 20DGRPL diets, respectively. The 10DGRPL and 20DGRPL diets met 110 and 100\% of the Lys requirements, respectively. Periods lasted 21 $\mathrm{d}$, with the last $3 \mathrm{~d}$ for data collection. Compared with cows fed the CON diet, cows fed diets with DDGS had a similar dry matter intake (DMI; $25.4 \pm 0.88 \mathrm{~kg} / \mathrm{d}$ ), milk yield $(30.7 \pm 1.67 \mathrm{~kg} / \mathrm{d})$, and composition, except for protein percentage, which was higher (3.15 vs. 3.21 $\pm 0.05 \%)$ and resulted in higher (0.94 vs. $1.00 \pm 0.05$ $\mathrm{kg} / \mathrm{d}$ ) protein yield by cows fed diets containing $20 \%$ DDGS. Unexpectedly, despite diets being formulated based on predicted DMI of $23.3 \mathrm{~kg} / \mathrm{d}$ and milk yield of $38.5 \mathrm{~kg} / \mathrm{d}$, cows had a greater DMI and lower milk yield across all treatments, which resulted in diets that were predicted by CPM Dairy to supply sufficient amounts of Lys $(140,118$, and $104 \%$ of requirement for the CON, 10DG, and 20DG diet, respectively) and consequently, supplementation with RPL did not have an effect on milk production or composition. Plasma concentration
\end{abstract}

Received April 8, 2013.

Accepted July 20, 2013

${ }^{1}$ Corresponding author: pkononoff $2 @ u n l . e d u$ of Lys decreased (11.8\%) as DDGS inclusion increased. For other essential AA, plasma concentrations of cows fed diets with DDGS were lower for Arg, His, and Val and greater for Leu and Met compared with cows fed the CON diet. Supplementation with RPL failed to decrease the plasma concentration of other essential AA, which provides support that Lys was not limiting.

Key words: dairy cow, distillers dried grains with solubles, rumen-protected lysine

\section{INTRODUCTION}

An increase in the availability and quality of distillers dried grains with solubles (DDGS) generated from the corn ethanol industry has promoted the development of research oriented toward finding the most effective ways to utilize this coproduct for milk production. This feed ingredient is characterized by a high concentration of CP (30\% of DM) and energy (2.26 Mcal of $\mathrm{NE}_{\mathrm{L}} /$ kg; Schingoethe et al., 2009), but low Lys concentration (1.86\% of RUP; Kelzer et al., 2010). Despite low levels of metabolizable Lys coming from DDGS, only a few studies (Owen and Larson, 1991; Kleinschmit et al., 2006; Mjoun et al., 2010b) have reported negative effects on milk protein when feeding DDGS. One potential reason for this is that in studies where milk protein did not change (Janicek et al., 2008; Zhang et al., 2010), the diets fed were high in CP concentration $(>18.0 \%$ of DM) and as a consequence, even if the dietary Lys concentration (\% of CP) was low, the total amount of Lys supplied to the cows met or exceeded the requirement. The feeding of high-CP diets may be problematic because of the excessive amount of $\mathrm{N}$ excreted by the cow into the environment (Frank and Swensson, 2002). As a result, a current need exists to evaluate the effect of feeding DDGS in diets not containing excessive amounts of CP.

Supplementation of rumen-protected AA has proven to be an effective route to supply limiting AA in dairy diets (NRC, 2001). However, information about supplementation of individual rumen-protected essential AA (EAA) is limited, except for Met (Patton, 2010). Lysine is cited as the most-limiting AA in diets that 
rely on corn-based ingredients (NRC, 2001); thus high inclusion of DDGS has not been favored in the field (Schwab, 2010). Supplementation of rumen-protected Lys (RPL) could be beneficial if Lys is limiting in diets with DDGS. The objective of this study was to evaluate the effects of feeding increasing amounts of DDGS in diets with or without the supplementation of an RPL product on milk yield and composition and plasma concentration of AA.

\section{MATERIALS AND METHODS}

\section{Animals and Experimental Treatments}

Twenty multiparous Holstein cows averaging $62 \pm 28$ DIM were assigned to one of four $5 \times 5$ Latin squares. Cows were blocked by milk yield and DIM and then treatments were randomly assigned to cows. Experimental periods lasted $21 \mathrm{~d}$, with $18 \mathrm{~d}$ for diet adaptation and $3 \mathrm{~d}$ for data collection. During the entire experiment, cows were housed in a tie-stall barn, fed once daily at $0900 \mathrm{~h}$ for ad libitum consumption to ensure at least $5 \%$ orts, and had continuous access to water. Additionally, cows were milked twice daily at $0700 \mathrm{~h}$ and $1900 \mathrm{~h}$. Animal care and experimental procedures were approved by the University of Nebraska-Lincoln Animal Care and Use Committee.

Diets comprised $53.3 \%$ forage and $46.7 \%$ concentrate (Table 1). Forages and cottonseed were premixed in a mixer wagon (Roto-Mix 312; Roto-Mix Inc., Dodge City, KS). In addition to the premixed forages and cottonseed, the specific concentrate of each diet was added into the Calan Data Ranger (American Calan Inc., Northwood, NH) to prepare the TMR. Diets were formulated using the Cornell-Penn-Miner (CPM) Dairy model (Boston et al., 2000) to be isoenergetic (2.59 Mcal of $\mathrm{ME} / \mathrm{kg}$ ) and isonitrogenous (16.4\% CP, DM basis) but were expected to provide a decreasing supply of MP-Lys. The latter was achieved by using DDGS to replace supplements higher in Lys. Dietary treatments were (1) control (CON; no DDGS), (2) 10\% DDGS (10DG), (3) 20\% DDGS (20DG), (4) 10\% DDGS plus RPL (10DGRPL), and (5) 20\% DDGS plus RPL (20DGRPL). Based on the CPM Dairy predictions, the CON diet was formulated to meet or exceed the requirements of a lactating dairy cow weighing $680 \mathrm{~kg}$, with a BCS of 3.00 , consuming $23.3 \mathrm{~kg}$ of feed daily, and producing $38.5 \mathrm{~kg}$ of milk daily with a composition of 3.0 and $3.5 \%$ of true protein and fat, respectively. In the CON diet, Lys was predicted to supply $6.86 \%$ of the MP and meet $117 \%$ of the MP-Lys requirements of the animals. Two more treatments containing increasing amounts of DDGS were formulated and Lys was predicted to supply 6.38 and $5.88 \%$ of the MP and meet
99 and $91 \%$ of the MP-Lys requirements of the animals (10DG and 20DG diets, respectively) and these 2 diets will be referred as unsupplemented diets. Two additional treatments (10DGRPL and 20DGRPL diets) were achieved by supplementing the 10DG and 20DG diets with an RPL product (AminoShure-L, L-Lysine 38\%; Balchem Encapsulates, New Hampton, NY) once daily at $60 \mathrm{~g} / \mathrm{d}$ via top dressing evenly over the TMR. Supplemented RPL was estimated to provide $14.6 \mathrm{~g}$ of MP-Lys; consequently, the 10DGRPL and 20DGRPL diets met 110 and $100 \%$ of the MP-Lys requirements of the animals. To ensure that all diets provided sufficient Met to avoid Met limiting potential milk protein responses by Lys supplementation, MetaSmart dry (Adisseo Inc., Antony, France) was added at a rate of $29 \mathrm{~g} / \mathrm{d}$ per cow.

\section{Experimental Measures and Sample Analysis}

Feed intake, orts, and milk production were recorded daily during the entire experiment. Dry matter concentrations of corn and alfalfa silages were determined weekly (microwave oven; Oetzel et al., 1993) and their respective inclusion in the diet was adjusted to account for DM variation. During the sampling period, forages, cottonseed, DDGS, concentrates, and TMR of the unsupplemented diets were sampled daily, refrigerated $\left(4^{\circ} \mathrm{C}\right)$, and composited at the end of each period. Composite samples of forages, cottonseed, DDGS, and concentrates were divided into 2 subsamples. One subsample from each composite was dried for $48 \mathrm{~h}$ at $55^{\circ} \mathrm{C}$ in a forced-air oven, subsequently ground with a Wiley Mill (1-mm screen; Arthur H. Thomas Co., Philadelphia, PA), and then reground with a Cyclotec 1093 mill (Tecator 1093; Foss Tecator AB, Höganäs, Sweden) to ensure uniform particle size. Ground samples were sent to the Experimental Station Chemical Laboratories, University of Missouri-Columbia and analyzed for AA (method 982.30 E; AOAC International, 2006) using a Hitachi L-8800 AA analyzer (Hitachi Co., Tokyo, Japan). The other subsample from each composite was sent to Cumberland Valley Analytical Services Inc. (Hagerstown, MD) for standard analysis, which included DM, N (Leco FP-528 Nitrogen Combustion Analyzer; Leco Corp., St. Joseph, MI), unavailable protein (haylages only), adjusted protein, soluble protein, degradable protein (calculated for forages only), ADF (method 973.18; AOAC International, 2000), NDF (Van Soest et al., 1991), ether extract (method 2003.05; AOAC International, 2006), ash (method 942.05; AOAC International, 2000), NFC, Ca, P, Mg, K, Na, Fe, Mn, $\mathrm{Zn}, \mathrm{Cu}$, and $\mathrm{pH}$ analysis (ensiled silages; Mettler DL12 Titrator; Mettler-Toledo Inc., Columbus, OH). Two subsamples from the TMR composites were taken. One 
$\mathrm{mL}$ ) were adjusted to account for the use of $15 \%$ sulfosalicylic acid.

\section{Statistical Analysis}

Analyses of animal responses and plasma concentration of AA were performed on period means of each cow. Data were analyzed as a replicated $5 \times 5$ Latin square using PROC MIXED of SAS (SAS Institute, 2008) based on the following model:

$$
\mathrm{Y}_{\mathrm{ijkl}}=\mu+\mathrm{S}_{\mathrm{i}}+\mathrm{p}_{\mathrm{j}}+\mathrm{c}_{\mathrm{k}: \mathrm{i}}+\mathrm{T}_{\mathrm{l}}+\varepsilon_{\mathrm{ijk} \mathrm{l}},
$$

where $Y_{\mathrm{ijkl}}=$ dependent variable, $\mu=$ overall mean, $\mathrm{S}_{\mathrm{i}}$ $=$ fixed effect of square $(3 \mathrm{df}), \mathrm{p}_{\mathrm{j}}=$ random effect of period $(4 \mathrm{df}), c_{\mathrm{k}: \mathrm{i}}=$ random effect of cow within square $(16 \mathrm{df}), \mathrm{T}_{1}=$ fixed effect of treatment $(4 \mathrm{df})$, and $\varepsilon_{\mathrm{ijkl}}$ $=$ residual error $(71 \mathrm{df})$. If a significant treatment $\mathrm{ef}-$ fect was observed $(P \leq 0.05)$, least squares means were separated using PDIFF. Treatment $\times$ square interaction was tested and removed because it was not significant $(P>0.10)$ in all the variables tested. At the end of first period, 1 cow on the CON treatment suffering from laminitis was replaced for a cow with similar DIM and milk production. No data were obtained from the cow suffering from laminitis; thus, the highest SEM are reported.

\section{RESULTS}

\section{Feeds and Diets Chemical Compositions}

The chemical composition of feeds and concentrates is presented in Table 2 and the chemical composition and particle size distribution of the CON and unsupplemented diets are presented in Table 3. Concentrations of NDF and fat increased and NFC decreased as inclusion of DDGS increased in the concentrates. The concentration of $\mathrm{CP}$ was similar across treatments, averaged $16.7 \%$ of DM, and differences in NDF, NFC, and fat concentrations reflected concentrates composition. The proportion of particles retained in the 3 screens and bottom pan of the Penn State Particle Separator was similar across treatments and averaged 14.6, 30.0, 33.2 , and $22.2 \%$ for the $>19-\mathrm{mm}, 8-\mathrm{mm}$, and $1.18-\mathrm{mm}$ screens, and bottom pan $(<1.18-\mathrm{mm})$, respectively.

The AA composition of feeds and concentrates is presented in Table 4 and the AA composition of the CON and unsupplemented diets is presented in Table 5 . Overall, differences in the AA composition of the concentrates were reflected in the AA composition of the diets. Specifically, an increase in the inclusion of DDGS from 0 to $20 \%$ of the diet DM resulted in an increase in the concentration of Ala (12.1\%), Cys (13.9\%), hy- droxyproline (Hyp; 25.5\%), Leu (10.4\%), Met (18.1\%), Orn $(28.6 \%)$, and Pro $(20.6 \%)$ and a decrease in the concentrations of Arg (11.3\%), Asp (17.7\%), His (7.4\%), Lys (18.5\%), and Trp (13.0\%).

\section{Animal Responses}

Treatment effects on DMI, BW, BCS, milk production, and composition are presented in Table 6 . Compared with the CON diet, inclusion of DDGS or supplementation with RPL did not affect DMI of the cows; however, within diets that contained DDGS, cows fed the 10DGRPL diet had the lowest DMI $(P=0.04)$. Nevertheless, milk yield, 3.5\% FCM, and feed conversion were similar across treatments, averaging $30.7 \pm$ $1.67,31.9 \pm 1.93$, and $1.26 \pm 0.07 \mathrm{~kg} / \mathrm{d}$, respectively. Treatments did not affect milk fat and lactose percentages but affected milk protein percentage $(P=0.03)$ and MUN $(P<0.01)$. Compared with cows fed the CON diet, cows fed diets containing DDGS produced milk with higher protein and MUN concentrations. One exception was the 20DGRPL diet, which had similar protein concentration as the CON diet. Fat yield was not affected by treatment and averaged $1.15 \pm 0.08$ $\mathrm{kg} / \mathrm{d}$. Cows fed diets with $20 \%$ DDGS had greater $(P$ $=0.04$ ) protein yield compared with those fed the CON diet but similar compared with those fed diets with $10 \%$ DDGS. Cows fed the 10DGRPL and 20DGRPL diets had similar milk yield and composition compared with those fed the respective unsupplemented diets. Cows fed the CON diet had a similar BW compared with those fed diets with DDGS, except for cows fed the 20DG diet, which were $10 \pm 3.0 \mathrm{~kg}$ heavier $(P=$ $0.04)$. Body condition score was similar among treatments and averaged $3.13 \pm 0.07$.

Treatment effects on AA intake are presented in Table 7. Intake of total EAA was similar between cows fed the CON diet and those fed the unsupplemented diets; however, specific intake of AA varied. Intake of Lys, Arg, His, and Trp decreased, whereas intake of Leu, Met, and Thr increased as inclusion of DDGS increased within the unsupplemented diets. Compared with their respective unsupplemented diet, intake of Lys was similar for cows fed the 10DGRPL diet and higher $(P<0.01)$ for cows fed the 20DGRPL diet.

\section{Plasma Concentration of $A A$}

Effects of diets on plasma concentrations of AA and urea sampled from the coccygeal artery are presented in Table 8. Compared with the cows fed the CON diet, plasma concentration of Lys was similar for cows fed the 10DG and 10DGRPL diets, lower $(P=0.01)$ for cows fed the 20DG diet, and tended $(P=0.06)$ to be 
Table 2. Chemical composition of feeds and concentrates

\begin{tabular}{|c|c|c|c|c|c|c|c|c|c|}
\hline \multirow[b]{2}{*}{ Item $^{2}$} & & \\
\hline & Corn silage & Alfalfa silage & Alfalfa hay & Brome hay & Cottonseed & DDGS & $\mathrm{CON}$ & $10 \mathrm{DG}$ & $20 \mathrm{DG}$ \\
\hline DM, $\%$ as fed & $34.3 \pm 3.03$ & $34.6 \pm 7.03$ & $84.4 \pm 0.37$ & $87.6 \pm 0.78$ & $89.8 \pm 0.95$ & $90.3 \pm 0.40$ & $88.7 \pm 0.89$ & $88.8 \pm 1.17$ & $89.3 \pm 0.31$ \\
\hline $\mathrm{CP}$ & $8.62 \pm 0.62$ & $15.8 \pm 1.21$ & $20.6 \pm 0.32$ & $8.12 \pm 1.18$ & $22.4 \pm 0.70$ & $28.2 \pm 0.38$ & $23.4 \pm 0.53$ & $23.6 \pm 0.25$ & $23.4 \pm 0.52$ \\
\hline $\mathrm{ADF}$ & $25.0 \pm 0.91$ & $37.1 \pm 1.73$ & $31.9 \pm 1.00$ & $43.9 \pm 2.94$ & $34.7 \pm 0.95$ & $11.1 \pm 1.18$ & $13.8 \pm 0.67$ & $14.7 \pm 1.60$ & $15.4 \pm 0.79$ \\
\hline NDF & $39.8 \pm 1.00$ & $44.3 \pm 2.27$ & $36.9 \pm 0.75$ & $68.4 \pm 3.16$ & $44.9 \pm 1.80$ & $31.6 \pm 1.08$ & $23.8 \pm 1.03$ & $28.3 \pm 2.30$ & $31.6 \pm 1.28$ \\
\hline Lignin & $3.14 \pm 0.30$ & $6.12 \pm 0.57$ & $6.97 \pm 0.28$ & $6.29 \pm 1.07$ & $9.08 \pm 0.97$ & $2.88 \pm 0.22$ & $2.36 \pm 0.50$ & $3.03 \pm 1.02$ & $3.59 \pm 0.95$ \\
\hline Sugar & $0.70 \pm 0.16$ & $1.28 \pm 0.56$ & $8.30 \pm 0.72$ & $7.06 \pm 2.25$ & $4.82 \pm 0.71$ & $3.60 \pm 0.71$ & $4.78 \pm 0.72$ & $4.26 \pm 0.23$ & $3.68 \pm 0.98$ \\
\hline Starch & $34.2 \pm 2.44$ & $3.00 \pm 1.27$ & $2.20 \pm 0.80$ & $1.98 \pm 0.89$ & $1.26 \pm 0.41$ & $5.58 \pm 0.84$ & $33.4 \pm 2.27$ & $24.5 \pm 1.24$ & $18.8 \pm 0.59$ \\
\hline Crude fat & $3.02 \pm 0.17$ & $2.65 \pm 0.28$ & $2.21 \pm 0.21$ & $1.86 \pm 0.23$ & $19.5 \pm 0.47$ & $11.7 \pm 0.67$ & $2.91 \pm 0.20$ & $5.04 \pm 0.29$ & $6.87 \pm 0.21$ \\
\hline $\mathrm{NFC}^{4}$ & $43.5 \pm 1.97$ & $19.7 \pm 3.54$ & $29.9 \pm 1.12$ & $12.2 \pm 4.88$ & $8.94 \pm 1.83$ & $23.0 \pm 0.91$ & $41.3 \pm 1.17$ & $34.2 \pm 2.20$ & $29.4 \pm 1.77$ \\
\hline Ash & $5.06 \pm 0.53$ & $17.5 \pm 2.09$ & $10.3 \pm 0.67$ & $9.41 \pm 0.95$ & $4.21 \pm 0.27$ & $5.41 \pm 0.65$ & $8.62 \pm 0.40$ & $8.80 \pm 0.38$ & $8.77 \pm 0.54$ \\
\hline $\mathrm{Ca}$ & $0.22 \pm 0.02$ & $1.28 \pm 0.11$ & $1.48 \pm 0.05$ & $0.32 \pm 0.04$ & $0.24 \pm 0.05$ & $0.03 \pm<0.01$ & $1.52 \pm 0.06$ & $1.44 \pm 0.11$ & $1.34 \pm 0.13$ \\
\hline $\mathrm{P}$ & $0.26 \pm 0.01$ & $0.37 \pm 0.09$ & $0.29 \pm 0.01$ & $0.26 \pm 0.02$ & $0.65 \pm 0.09$ & $0.93 \pm 0.04$ & $0.58 \pm 0.03$ & $0.58 \pm 0.01$ & $0.61 \pm 0.02$ \\
\hline $\mathrm{Mg}$ & $0.16 \pm 0.01$ & $0.38 \pm 0.02$ & $0.23 \pm 0.00$ & $0.11 \pm 0.01$ & $0.41 \pm 0.04$ & $0.34 \pm 0.02$ & $0.52 \pm 0.05$ & $0.52 \pm 0.03$ & $0.56 \pm 0.04$ \\
\hline $\mathrm{K}$ & $1.23 \pm 0.05$ & $5.19 \pm 1.12$ & $3.19 \pm 0.18$ & $2.24 \pm 0.25$ & $1.27 \pm 0.10$ & $1.35 \pm 0.05$ & $1.34 \pm 0.03$ & $1.39 \pm 0.05$ & $1.29 \pm 0.03$ \\
\hline $\mathrm{S}$ & $0.13 \pm 0.01$ & $0.26 \pm 0.03$ & $0.23 \pm 0.05$ & $0.13 \pm 0.02$ & $0.26 \pm 0.02$ & $0.99 \pm 0.04$ & $0.27 \pm 0.01$ & $0.43 \pm 0.01$ & $0.56 \pm 0.03$ \\
\hline $\mathrm{Na}$ & $0.01 \pm 0.00$ & $0.03 \pm 0.01$ & $0.02 \pm 0.00$ & $0.01 \pm 0.00$ & $0.04 \pm 0.02$ & $0.23 \pm<0.01$ & $0.77 \pm 0.08$ & $0.81 \pm 0.05$ & $0.82 \pm 0.06$ \\
\hline $\mathrm{Cl}$ & $0.17 \pm 0.01$ & $0.26 \pm 0.03$ & $0.17 \pm 0.07$ & $0.60 \pm 0.13$ & $0.08 \pm 0.02$ & $0.16 \pm 0.06$ & $0.72 \pm 0.07$ & $0.71 \pm 0.08$ & $0.69 \pm 0.06$ \\
\hline $\mathrm{Fe}, \mathrm{mg} / \mathrm{kg}$ & $109 \pm 19.5$ & $1,140 \pm 662$ & $322 \pm 54.8$ & $336 \pm 294.7$ & $76.2 \pm 16.3$ & $71.2 \pm 4.32$ & $504 \pm 68.7$ & $377 \pm 49.6$ & $307 \pm 26.6$ \\
\hline $\mathrm{Mn}, \mathrm{mg} / \mathrm{kg}$ & $27.4 \pm 1.82$ & $74.2 \pm 25.15$ & $48.0 \pm 4.69$ & $48.0 \pm 5.24$ & $23.2 \pm 5.07$ & $15.8 \pm 0.45$ & $118 \pm 12.9$ & $122 \pm 10.9$ & $142 \pm 18.1$ \\
\hline $\mathrm{Zn}, \mathrm{mg} / \mathrm{kg}$ & $38.2 \pm 3.70$ & $40.8 \pm 12.76$ & $21.2 \pm 2.39$ & $28.2 \pm 9.81$ & $44.4 \pm 5.81$ & $70.8 \pm 5.26$ & $189 \pm 9.15$ & $182 \pm 8.82$ & $205 \pm 29.1$ \\
\hline $\mathrm{Cu}, \mathrm{mg} / \mathrm{kg}$ & $8.60 \pm 0.55$ & $10.4 \pm 0.89$ & $10.0 \pm 0.71$ & $8.80 \pm 1.64$ & $10.4 \pm 1.34$ & $1.60 \pm 0.55$ & $55.8 \pm 5.93$ & $56.0 \pm 3.61$ & $57.2 \pm 8.04$ \\
\hline $\mathrm{pH}$ & $4.00 \pm 0.07$ & $4.82 \pm 0.47$ & - & - & $=$ & - & - & - & - \\
\hline
\end{tabular}

${ }^{1}$ Values $(\mathrm{n}=5)$ are expressed as mean \pm SD.

${ }^{2}$ Values are expressed in percentage of DM, unless otherwise noted.

${ }^{3} \mathrm{CON}=$ control diet containing no distillers dried grains with solubles (DDGS); 10DG and 20DG = diets containing DDGS at 10 and $20 \%$ of DM, respectively, with no rumenprotected Lys.

${ }^{4} \mathrm{NFC}=100-(\% \mathrm{NDF}+\% \mathrm{CP}+\%$ fat $+\%$ ash $)$. 
Table 3. Chemical composition (means \pm SD; \% of DM, unless otherwise noted) and particle size distribution of the control and unsupplemented diets

\begin{tabular}{|c|c|c|c|}
\hline \multirow[b]{2}{*}{ Item } & \multicolumn{3}{|c|}{$\operatorname{Diet}^{1}$} \\
\hline & $\mathrm{CON}$ & $10 \mathrm{DG}$ & $20 \mathrm{DG}$ \\
\hline \multicolumn{4}{|l|}{ Composition } \\
\hline DM & $52.3 \pm 0.83$ & $52.2 \pm 0.97$ & $52.3 \pm 0.89$ \\
\hline $\mathrm{CP}$ & $16.7 \pm 0.19$ & $16.8 \pm 0.24$ & $16.7 \pm 0.30$ \\
\hline $\mathrm{RDP}, \%$ of $\mathrm{CP}$ & 61.1 & 63.7 & 61.6 \\
\hline RUP, $\%$ of CP & 38.9 & 36.3 & 38.4 \\
\hline $\mathrm{ADF}$ & $22.8 \pm 0.66$ & $23.2 \pm 0.77$ & $23.5 \pm 0.61$ \\
\hline $\mathrm{NDF}$ & $34.8 \pm 0.71$ & $36.8 \pm 1.23$ & $38.2 \pm 0.47$ \\
\hline Lignin & $3.57 \pm 0.36$ & $3.87 \pm 0.61$ & $4.11 \pm 0.62$ \\
\hline Sugar & $3.36 \pm 0.42$ & $3.13 \pm 0.29$ & $2.88 \pm 0.59$ \\
\hline Starch & $26.7 \pm 0.37$ & $22.9 \pm 0.42$ & $20.3 \pm 0.70$ \\
\hline Crude fat & $3.22 \pm 0.23$ & $4.15 \pm 0.26$ & $4.95 \pm 0.25$ \\
\hline $\mathrm{NFC}^{2}$ & $37.0 \pm 0.97$ & $33.9 \pm 1.43$ & $31.8 \pm 0.89$ \\
\hline Ash & $8.30 \pm 0.27$ & $8.38 \pm 0.20$ & $8.37 \pm 0.29$ \\
\hline $\mathrm{Ca}$ & $0.96 \pm 0.01$ & $0.93 \pm 0.06$ & $0.88 \pm 0.06$ \\
\hline $\mathrm{P}$ & $0.42 \pm 0.02$ & $0.42 \pm 0.01$ & $0.44 \pm 0.01$ \\
\hline $\mathrm{Mg}$ & $0.35 \pm 0.03$ & $0.34 \pm 0.01$ & $0.36 \pm 0.02$ \\
\hline $\mathrm{K}$ & $1.81 \pm 0.09$ & $1.83 \pm 0.08$ & $1.79 \pm 0.10$ \\
\hline $\mathrm{S}$ & $0.21 \pm 0.01$ & $0.28 \pm 0.01$ & $0.34 \pm 0.02$ \\
\hline $\mathrm{Na}$ & $0.35 \pm 0.04$ & $0.37 \pm 0.02$ & $0.37 \pm 0.03$ \\
\hline $\mathrm{Cl}$ & $0.45 \pm 0.03$ & $0.44 \pm 0.03$ & $0.43 \pm 0.04$ \\
\hline $\mathrm{ME},{ }^{3} \mathrm{Mcal} / \mathrm{kg}$ & 2.52 & 2.51 & 2.50 \\
\hline MP-Lys, ${ }^{3} \mathrm{~g} / \mathrm{d}$ & 195 & 171 & 156 \\
\hline MP-Met, ${ }^{3} \mathrm{~g} / \mathrm{d}$ & 65 & 66 & 66 \\
\hline \multicolumn{4}{|c|}{ Particle size distribution ${ }^{4}$} \\
\hline$>19.0 \mathrm{~mm}$ & $12.6 \pm 7.15$ & $15.3 \pm 10.7$ & $16.0 \pm 7.11$ \\
\hline $8.0-19.0 \mathrm{~mm}$ & $31.0 \pm 3.70$ & $29.8 \pm 1.16$ & $29.1 \pm 3.31$ \\
\hline $1.18-8.0 \mathrm{~mm}$ & $36.3 \pm 4.00$ & $30.3 \pm 0.64$ & $33.1 \pm 1.64$ \\
\hline$<1.18 \mathrm{~mm}$ & $20.1 \pm 8.06$ & $24.6 \pm 11.4$ & $21.8 \pm 8.91$ \\
\hline
\end{tabular}

${ }^{1} \mathrm{CON}=$ control diet containing no distillers dried grains with solubles (DDGS); 10DG and 20DG = diets containing DDGS at 10 and $20 \%$ of DM, respectively, with no rumen-protected Lys.

${ }^{2} \mathrm{NFC}=100-(\% \mathrm{NDF}+\% \mathrm{CP}+\%$ fat $+\%$ ash $)$.

${ }^{3}$ Estimation based on CPM Dairy (v3.0; Boston et al., 2000) using assayed feed analyses and observed DMI for each treatment. MP-Lys = metabolizable Lys supply and MP-Met = metabolizable Met supply.

${ }^{4}$ Proportion (as fed) of TMR retained on each screen of the Penn State Particle Separator (The Pennsylvania State University, University Park).

lower for cows fed the 20DGRPL diet. For other EAA, plasma concentrations of cows fed diets with DDGS were lower for His $(P=0.03)$ and $\operatorname{Val}(P=0.01)$ and greater for Met $(P<0.01)$ and Leu $(P=0.01)$ compared with cows fed the CON diet. Plasma concentration of Arg was lower $(P=0.04)$ for cows fed diets with $20 \%$ DDGS compared with those fed diets with 10\% DDGS and similar to cows fed the CON diet. Supplementation with RPL did not increase plasma concentration of Lys and had no effect on plasma concentration of other AA. Cows fed diets with DDGS had a tendency $(P=$ $0.07)$ to have a higher plasma concentration of urea compared with those fed the CON $\operatorname{diet}(308.2$ vs. 281.1 $\pm 22.3 \mu \mathrm{g} / \mathrm{mL}$, respectively).

\section{DISCUSSION}

Based on the indirect dose-response approach, the NRC (2001) suggests that the concentration of Lys in MP to maximize milk protein percentage and yield should be $7.2 \%$. The low concentration of Lys in the RUP of DDGS indicates that excess RUP will have to be fed to formulate diets that could match as closely as possible the NRC recommendation, thus increasing dietary CP concentration. However, Anderson et al. (2006) found no effects on animal performance when feeding diets containing 0 or $20 \%$ DDGS without a gross oversupply of CP (17\%). This demonstrates that diets can effectively be formulated to contain high amounts of DDGS and yet still supply adequate amounts of Lys. Limited information exists on the effects of low-CP diets containing high inclusion of DDGS on milk yield and composition. If Lys is limiting in these types of diets, supplementation with RPL may be used to provide additional Lys to meet the animal requirements. The present study evaluated the concept of supplementing relatively low-CP diets (16.7\% of DM) with increasing amounts of DDGS to determine if milk yield and com- 
Table 4. Amino acid composition of feeds and concentrates ${ }^{1}$

Feed

Feed

Corn silage

Alfalfa silage

Alfalfa hay

Brome hay

Cottonseed

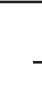

\begin{tabular}{ccc}
\multicolumn{3}{c}{ Concentrate $^{2}$} \\
\hline CON & 10DG & \multicolumn{1}{c}{$20 \mathrm{DG}$} \\
\hline & & \\
$5.36 \pm 0.29$ & $5.16 \pm 0.26$ & $4.44 \pm 0.21$ \\
$2.67 \pm 0.09$ & $2.32 \pm 0.10$ & $2.30 \pm 0.11$ \\
$3.59 \pm 0.17$ & $3.52 \pm 0.32$ & $3.43 \pm 0.09$ \\
$7.90 \pm 0.21$ & $8.03 \pm 0.28$ & $8.61 \pm 0.39$ \\
$5.09 \pm 0.29$ & $4.51 \pm 0.29$ & $3.74 \pm 0.13$ \\
$1.22 \pm 0.10$ & $1.46 \pm 0.14$ & $1.50 \pm 0.08$ \\
$4.48 \pm 0.18$ & $4.19 \pm 0.16$ & $4.08 \pm 0.22$ \\
$3.26 \pm 0.17$ & $3.37 \pm 0.09$ & $3.27 \pm 0.18$ \\
$0.89 \pm 0.03$ & $0.83 \pm 0.06$ & $0.70 \pm 0.06$ \\
$4.70 \pm 0.19$ & $4.18 \pm 0.41$ & $4.25 \pm 0.11$ \\
$39.2 \pm 1.42$ & $37.6 \pm 1.86$ & $36.3 \pm 1.46$ \\
& & \\
$4.66 \pm 0.12$ & $4.99 \pm 0.16$ & $5.51 \pm 0.19$ \\
$9.20 \pm 0.44$ & $8.06 \pm 0.43$ & $6.72 \pm 0.14$ \\
$1.38 \pm 0.03$ & $1.52 \pm 0.05$ & $1.56 \pm 0.10$ \\
$14.25 \pm 0.83$ & $13.84 \pm 0.86$ & $12.72 \pm 0.33$ \\
$4.30 \pm 0.18$ & $4.28 \pm 0.19$ & $4.12 \pm 0.12$ \\
$0.14 \pm 0.03$ & $0.19 \pm 0.04$ & $0.23 \pm 0.05$ \\
$0.53 \pm 0.30$ & $0.64 \pm 0.40$ & $0.73 \pm 0.52$ \\
$0.08 \pm 0.02$ & $0.08 \pm<0.01$ & $0.10 \pm 0.02$ \\
$4.87 \pm 0.10$ & $5.62 \pm 0.17$ & $5.91 \pm 0.37$ \\
$3.86 \pm 0.38$ & $3.89 \pm 0.19$ & $3.73 \pm 0.16$ \\
$0.39 \pm 0.08$ & $0.40 \pm 0.06$ & $0.32 \pm 0.05$ \\
$3.11 \pm 0.15$ & $3.28 \pm 0.14$ & $3.26 \pm 0.17$ \\
\hline & &
\end{tabular}

Ess

Arg
His
Ile

Ile

Leu

Lys
Met

Phe

Thr

Trp

Val TEAA $^{3}$

1.57

$2.32 \pm 0.21$

$4.77 \pm 0.28$

$3.78 \pm 0.37$

$10.13 \pm 0.83$

$4.10 \pm 0.35$

$1.84 \pm 0.10$

$1.32 \pm 0.14$

$2.59 \pm 0.19$

$3.21 \pm 0.36$

$3.05 \pm 0.24$

$7.75 \pm 0.24$

$6.16 \pm 0.57$

$5.53 \pm 0.44$

$\begin{array}{ll}8.48 \pm 0.58 & 6.67 \pm 0.79 \\ 1.95 \pm 0.49 & 3.13 \pm 0.68\end{array}$

$1.95 \pm 0.49$

$3.13 \pm 0.68$

$5.22 \pm 0.32$

$3.43 \pm 0.48$

$4.37 \pm 0.33$

$1.63 \pm 0.11$

$1.35 \pm 0.17$

$1.47 \pm 0.15$

$4.96 \pm 0.33$

$3.85 \pm 0.52$

$5.10 \pm 0.19-3.82 \pm 0.35$
$4.26 \pm 0.35-3.40 \pm 0.25$

$3.82 \pm 0.35$

$3.24 \pm 0.27$

$2.77 \pm 0.60$

$4.26 \pm 0.35$

$3.40 \pm 0.25$

$3.01 \pm 0.29$

$\begin{array}{lll}1.00 \pm 0.10 & 0.64 \pm 0.09 & 0.76 \pm 0.13\end{array}$

$\begin{array}{lll}0.47 \pm 0.03 & 0.56 \pm 0.09 & 1.00 \pm 0.10 \\ 4.35 \pm 0.24 & 5.13 \pm 0.51 & 5.69 \pm 0.19\end{array}$

$4.27 \pm 0.53$

$4.20 \pm 0.37$

$31.1 \pm 2.40$

$41.8 \pm 1.68$

$28.8 \pm 2.72$

$40.1 \pm 3.22$

Nonessential AA, ${ }^{4} \%$ of $\mathrm{CP}$

Ala

$28.8 \pm 2.72$

$9.55 \pm 1.69$

$5.46 \pm 0.33$

$9.11 \pm 0.32$
$4.15 \pm 0.73$

$4.85 \pm 0.81$

$5.46 \pm 0.33$
$11.53 \pm 1.10$

$4.95 \pm 0.49$

$3.70 \pm 0.39$

$1.11 \pm 0.11$

$0.83 \pm 0.13$

$\begin{aligned} 1.18 & \pm 0.09\end{aligned}$

$6.68 \pm 0.51$

$8.50 \pm 0.88$

$8.50 \pm 0.88$
$1.61 \pm 0.17$

$9.41 \pm 0.65$

$\begin{array}{rrr}\text { Glu } & 10.04 \pm 1.57 & 5.75 \pm 0.66 \\ \text { Gly } & 3.65 \pm 0.14 & 4.06 \pm 0.20\end{array}$

$\begin{array}{ll}\text { Gly } & 3.65 \pm 0.14 \\ \text { Hyl } & 4.23 \pm 0.90\end{array}$

Hyp $\quad 0.31 \pm 0.17$

Orn $\quad 44.46 \pm 1.86$

$\begin{array}{ll}\text { Pro } & 5.84 \pm 0.37 \\ \text { Ser } & 2.08 \pm 0.26\end{array}$

$5.53 \pm 1.98$

$4.87 \pm 0.21$

$8.27 \pm 0.34$

$1.61 \pm 0.17$
$1.82 \pm 0.09$

$1.82 \pm 0.09$

$0.61 \pm 0.06$

$1.08 \pm 0.07$

$4.22 \pm 0.37$

$3.90 \pm 0.42$

$0.35 \pm 0.20$

$0.87 \pm 0.07$

$0.35 \pm 0.07$

$0.14 \pm 0.04$

$0.10 \pm<0.01$

$0.56 \pm 0.32$

$2.30 \pm 0.45$

$0.59 \pm 0.14 \quad 0.45 \pm 0.13$

$3.68 \pm 0.63$

$0.05 \pm<0.01$

$4.19 \pm 0.27$

$3.52 \pm 0.28$

$\begin{array}{lll}7.47 \pm 0.89 \pm 0.63 & 2.97 \pm 0.18 & 3.62 \pm 0.61 \\ 0.42 \pm 0.14 & 0.67 \pm 0.15 & 0.18 \pm 0.05\end{array}$

$0.18 \pm 0.05$

$2.01 \pm 0.21 \quad 3.33 \pm 0.24$

$2.06 \pm 0.14$

$2.63 \pm 0.24$

${ }^{\mathrm{I}}$ Values $(\mathrm{n}=5)$ are expressed as mean $\pm \mathrm{SD}$

${ }^{2} \mathrm{CON}=$ control diet containing no distillers dried grains with solubles (DDGS); 10DG and 20DG = diets containing DDGS at 10 and $20 \%$ of DM, respectively, with no rumenprotected Lys.

Total essential AA (Arg, His, Ile, Leu, Lys, Met, Phe, Thr, Trp, and Val).

${ }^{4} \mathrm{Hyl}=$ hydroxylysine; Hyp = hydroxyproline. 
Table 5. Amino acid composition of the control and unsupplemented $\operatorname{diets}^{1}$

\begin{tabular}{|c|c|c|c|}
\hline \multirow[b]{2}{*}{ Item } & \multicolumn{3}{|c|}{$\operatorname{Diet}^{2}$} \\
\hline & $\mathrm{CON}$ & $10 \mathrm{DG}$ & $20 \mathrm{DG}$ \\
\hline \multicolumn{4}{|c|}{ Essential AA, $\%$ of CP } \\
\hline Arg & $4.51 \pm 0.23$ & $4.40 \pm 0.16$ & $4.00 \pm 0.17$ \\
\hline His & $2.15 \pm 0.07$ & $1.98 \pm 0.08$ & $1.99 \pm 0.12$ \\
\hline Ile & $3.51 \pm 0.16$ & $3.48 \pm 0.21$ & $3.46 \pm 0.10$ \\
\hline Leu & $7.41 \pm 0.47$ & $7.74 \pm 0.26$ & $8.18 \pm 0.28$ \\
\hline Lys & $4.27 \pm 0.18$ & $3.89 \pm 0.18$ & $3.48 \pm 0.14$ \\
\hline Met & $1.27 \pm 0.10$ & $1.46 \pm 0.10$ & $1.50 \pm 0.06$ \\
\hline Phe & $4.16 \pm 0.22$ & $4.03 \pm 0.14$ & $3.99 \pm 0.20$ \\
\hline Thr & $3.08 \pm 0.17$ & $3.20 \pm 0.08$ & $3.19 \pm 0.09$ \\
\hline $\operatorname{Trp}$ & $0.77 \pm 0.05$ & $0.74 \pm 0.05$ & $0.67 \pm 0.04$ \\
\hline Val & $4.60 \pm 0.18$ & $4.32 \pm 0.22$ & $4.39 \pm 0.09$ \\
\hline TEAA $^{3}$ & $35.7 \pm 1.46$ & $35.2 \pm 1.24$ & $34.8 \pm 1.11$ \\
\hline \multicolumn{4}{|c|}{ Nonessential $\mathrm{AA},{ }^{4} \%$ of $\mathrm{CP}$} \\
\hline Ala & $5.64 \pm 0.62$ & $5.96 \pm 0.29$ & $6.32 \pm 0.30$ \\
\hline Asp & $7.84 \pm 0.31$ & $7.21 \pm 0.25$ & $6.45 \pm 0.17$ \\
\hline Cys & $1.22 \pm 0.04$ & $1.34 \pm 0.06$ & $1.39 \pm 0.09$ \\
\hline Glu & $11.90 \pm 0.80$ & $12.10 \pm 0.58$ & $11.58 \pm 0.46$ \\
\hline Gly & $4.07 \pm 0.18$ & $4.12 \pm 0.13$ & $4.07 \pm 0.07$ \\
\hline Hyl & $1.37 \pm 0.38$ & $1.37 \pm 0.25$ & $1.36 \pm 0.33$ \\
\hline Нyp & $0.51 \pm 0.22$ & $0.57 \pm 0.30$ & $0.64 \pm 0.35$ \\
\hline Orn & $0.14 \pm 0.33$ & $0.16 \pm 0.06$ & $0.18 \pm 0.05$ \\
\hline Pro & $4.71 \pm 0.33$ & $5.39 \pm 0.10$ & $5.68 \pm 0.18$ \\
\hline Ser & $3.30 \pm 0.32$ & $3.41 \pm 0.12$ & $3.30 \pm 0.10$ \\
\hline Tau & $0.41 \pm 0.10$ & $0.43 \pm 0.07$ & $0.38 \pm 0.06$ \\
\hline Tyr & $2.68 \pm 0.16$ & $2.81 \pm 0.13$ & $2.83 \pm 0.15$ \\
\hline
\end{tabular}

${ }^{1}$ Values $(\mathrm{n}=5)$ are expressed as mean $\pm \mathrm{SD}$.

${ }^{2} \mathrm{CON}=$ control diet containing no distillers dried grains with solubles (DDGS); 10DG and 20DG $=$ diets containing DDGS at 10 and $20 \%$ of DM, respectively, with no rumen-protected Lys.

${ }^{3}$ Total essential AA (Arg, His, Ile, Leu, Lys, Met, Phe, Thr, Trp, and Val).

${ }^{4} \mathrm{Hyl}=$ hydroxylysine; Hyp $=$ hydroxyproline.

Table 6. Effect of diets on intake, BW, BCS, milk production, and composition

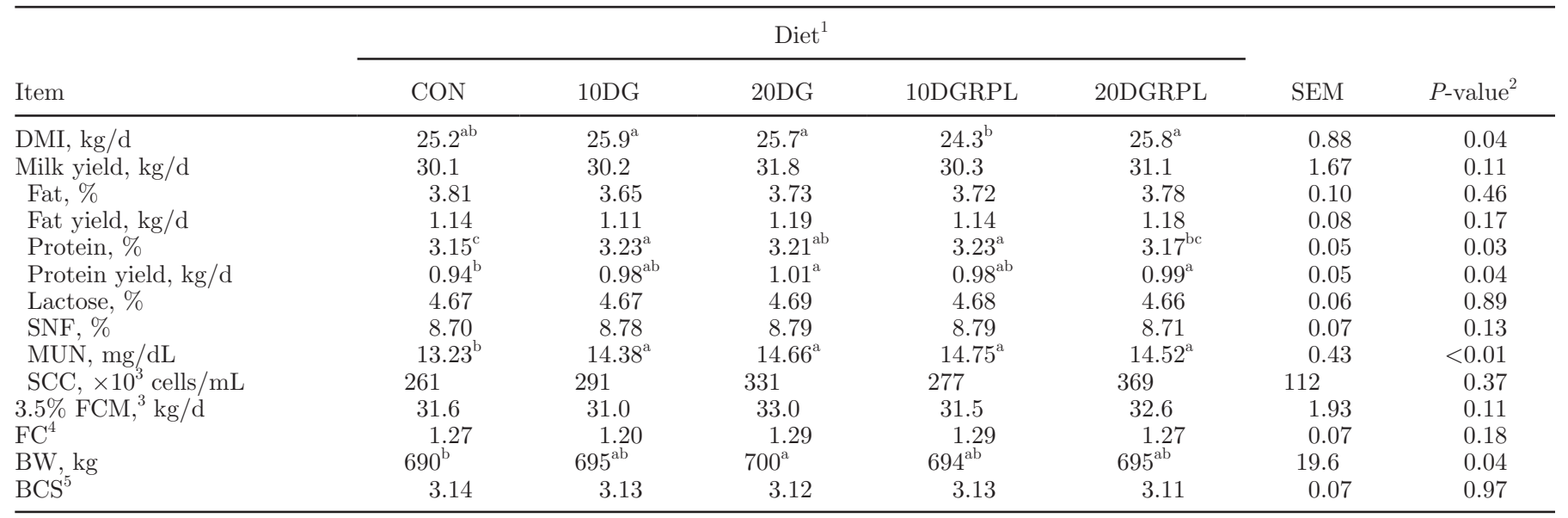

${ }^{\mathrm{a}-\mathrm{c}}$ Means within a row with different superscripts differ $(P \leq 0.05)$.

${ }^{1} \mathrm{CON}=$ control diet containing no distillers dried grains with solubles (DDGS); 10DG and 20DG = diets containing DDGS at 10 and $20 \%$ of DM, respectively, with no rumen-protected Lys (RPL); 10DGRPL and 20DGRPL = diets containing DDGS at 10 or $20 \%$ of DM, respectively, plus RPL.

${ }^{2}$ Treatment effect.

${ }^{3} \mathrm{FCM}=[$ milk fat $(\mathrm{kg} / \mathrm{d}) \times 16.218]+[$ milk yield $(\mathrm{kg} / \mathrm{d}) \times 0.4324]$.

${ }^{4}$ Feed conversion $=$ milk yield $/$ DMI.

${ }^{5}$ Cow BCS was determined on a 1 to 5 scale according to Wildman et al. (1982). 
Table 7. Effect of diets on AA intake

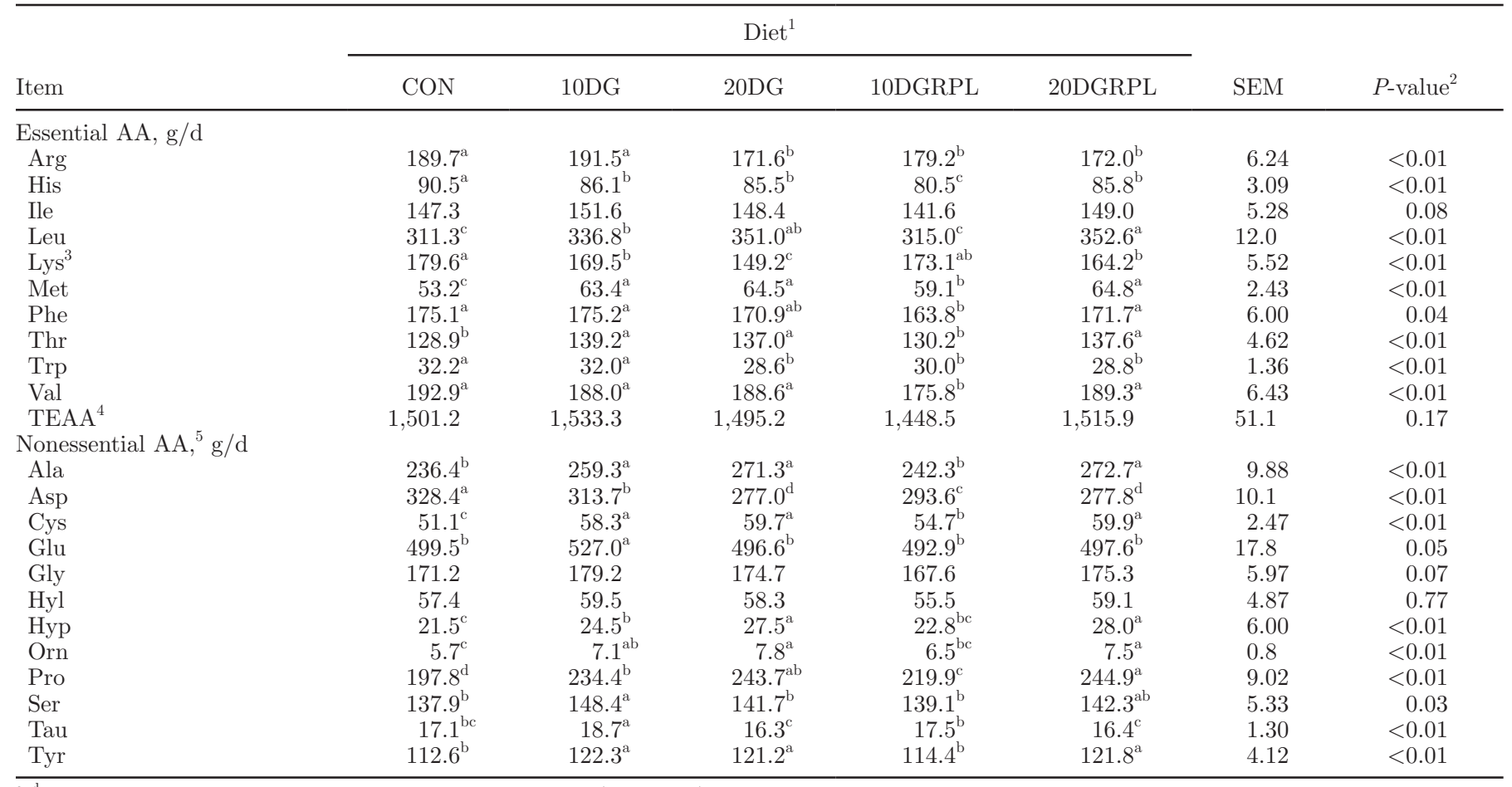

${ }^{\mathrm{a}-\mathrm{d}}$ Means within a row with different superscripts differ $(P \leq 0.05)$.

${ }^{1} \mathrm{CON}=$ control diet containing no distillers dried grains with solubles (DDGS); 10DG and 20DG = diets containing DDGS at 10 and $20 \%$ of DM, respectively, with no rumen-protected Lys (RPL); 10DGRPL and 20DGRPL = diets containing DDGS at 10 and $20 \%$ of DM, respectively, plus RPL.

${ }^{2}$ Treatment effect.

${ }^{3}$ 10DGRPL and 20DGRPL include Lys from the RPL product.

${ }^{4}$ Total essential AA (Arg, His, Ile, Leu, Lys, Met, Phe, Thr, Trp, and Val).

${ }^{5} \mathrm{Hyl}=$ hydroxylysine; Hyp = hydroxyproline

position could be maintained and also if supplementing these diets with RPL would enhance yields of milk and protein. Plasma concentration of AA were used as a tool to evaluate the AA status of the cows.

\section{Feed and Diet Chemical Compositions}

The chemical composition of cottonseed and of all forages fell within the normal ranges (NRC, 2001) except for the elevated ash and low CP concentrations of alfalfa silage. Alfalfa silage fed during this study had an $\mathrm{Fe}$ concentration almost 3 times higher than normal (NRC, 2001), which suggests that soil contamination (Hansen and Spears, 2009) increased the concentration of ash and diluted the concentration of CP. Nevertheless, the CP concentration of the assayed diets (averaged $16.7 \%$ of DM) closely resembled that of the formulated diets (16.4\% of DM), as alfalfa silage was included in the same proportion across treatments and the variation between assayed and expected $\mathrm{CP}$ concentration of the rest of the dietary ingredients was minor. The chemical composition of DDGS resembled modern corn-milling practices (Kelzer et al., 2010). Concentrations of CP, P, and S of DDGS were 28.2, 0.93 , and $0.99 \%$ of DM, respectively.

Compared with the recommendations of particle size distribution among the different screens (Heinrichs and Kononoff, 2002), a slightly higher (6.6\%) deviation from recommendations was observed for the $>19-\mathrm{mm}$ screen across treatments; however, the magnitude of the difference was small and the effects on production responses were most likely negligible. Across treatments, the concentration of total EAA was similar (averaged $35.2 \%$ ) and the AA profile reflected the AA profile of the concentrates, as the proportion of forage and the specific inclusion rate of each forage source was consistent across treatments.

\section{Animal Responses}

Cows fed diets containing DDGS had a similar DMI as those fed the CON diet. Past studies have found no 
Table 8. Effect of diets on plasma concentrations of AA and urea from the coccygeal artery

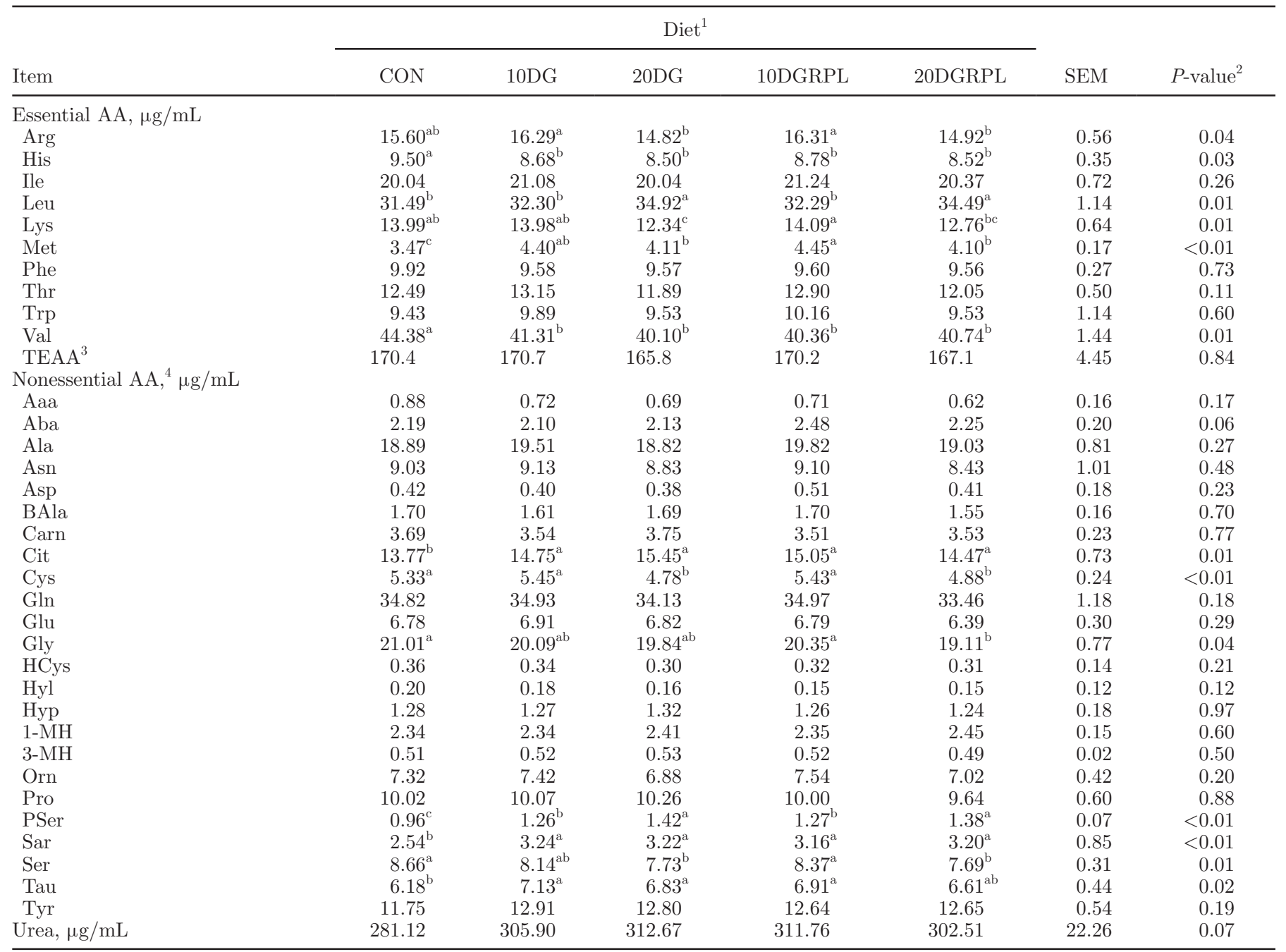

${ }^{\mathrm{a}-\mathrm{c}}$ Means within a row with different superscripts differ $(P \leq 0.05)$.

${ }^{1} \mathrm{CON}=$ control diet containing no distillers dried grains with solubles (DDGS); 10DG and 20DG = diets containing DDGS at 10 and $20 \%$ of DM, respectively, with no rumen-protected Lys (RPL); 10DGRPL and 20DGRPL = diets containing DDGS at 10 and $20 \%$ of DM, respectively, plus RPL.

${ }^{2}$ Treatment effect.

${ }^{3}$ Total essential AA (Arg, His, Ile, Leu, Lys, Met, Phe, Thr, Trp, and Val).

${ }^{4} \mathrm{Aaa}=\alpha$-aminoadipic acid; Aba $=\alpha$-amino- $n$-butyric acid; Asn $=$ asparagine; $\mathrm{BAla}=\beta$-alanine; Carn $=$ carnosine; Cit $=$ citrulline; Gln $=$ glutamine; HCys = homocystine; 1-MH = 1-methylhistidine; 3-MH = 3-methylhistidine; PSer = phosphoserine; Sar = sarcosine.

effects on DMI by including DDGS up to $20 \%$ of the diet DM (Kleinschmit et al., 2006; Janicek et al., 2008). Observed intake was actually higher than anticipated (CPM Dairy estimated at $23.3 \mathrm{~kg} / \mathrm{d}$ ) by about $1 \mathrm{~kg} / \mathrm{d}$ for cows fed the 10DGRPL diet and, on average, 2.5 $\mathrm{kg} / \mathrm{d}$ higher for cows fed the other diets containing DDGS. Similar milk and FCM yields between cows fed the CON diet and cows fed the diets containing DDGS is consistent with the results of Christen et al. (2010). When diets that contained DDGS were formulated to have a higher energy content (Kleinschmit et al., 2006) or cows consumed more of the diets containing DDGS
(Janicek et al., 2008), milk production has been reported to be greater compared with the diet that did not include DDGS. In the present study, diets were formulated to be isoenergetic and DMI by the cows fed diets containing DDGS and those fed the CON diet was similar. Across treatments, milk yield was lower than expected $(38.5 \mathrm{~kg} / \mathrm{d})$ throughout the entire experiment and averaged $34.1,32.9,30.6,29.1$, and $26.8 \mathrm{~kg} / \mathrm{d}$ from period 1 to 5 , respectively. Overall and surprisingly so, milk yield was $7.8 \mathrm{~kg} / \mathrm{d}$ lower than anticipated across treatments. As observed, milk fat percentage is frequently not affected when diets contain DDGS up to 
$20 \%$ of the DM (Kleinschmit et al., 2006; Hubbard et al., 2009). In this study, similar milk yield and fat percentage across treatments resulted in similar fat yield. Despite the fact that plasma concentration of Lys decreased as DDGS inclusion increased in the diets, milk protein percentage and yield increased. Mjoun et al. (2010b) reported similar observations and attributed the positive effects on milk protein to a greater uptake of Met and branched-chain AA by the mammary gland of cows fed the diet with DDGS compared with the ones fed the diet without DDGS. In the present study, we could not determine if the latter occurred because blood was only sampled from the coccygeal artery and to estimate mammary uptake, the arteriovenous differences in plasma concentration of AA and mammary plasma flow are required. Inclusion of DDGS increased MUN concentration. The plasma concentration of the AA 3-methylhistidine has been used as an indicator of muscle protein mobilization by the cow (Sawada et al., 2012). Across diets, plasma concentration of 3-methylhistidine was similar, which indicates that the difference in MUN values was not related to the disposal of $\mathrm{N}$ coming from the breakdown of muscle protein. In diets with DDGS, a portion of ground corn was replaced by DDGS, resulting in a lower starch and higher NDF content compared with the CON diet. Hristov et al. (2005) observed that starch from corn increased rumen ammonia utilization, which resulted in a decrease in MUN concentration. Thus, the increase in MUN concentration with the inclusion of DDGS may be related to a lower supply of starch.

Others (Blauwiekel et al., 1997; Robinson et al., 2011) have also reported no effect on DMI by the cows when fed diets supplemented with RPL. Responses in milk yield when diets estimated to be deficient in Lys were supplemented with RPL have been inconsistent. Swanepoel et al. (2010) reported no effect on milk yield when feeding a diet with RPL that supplied an estimated $10 \mathrm{~g}$ of additional intestinally absorbable Lys compared with the control diet. In contrast, Robinson et al. (2011) reported an increase in milk yield of around $2 \mathrm{~kg} / \mathrm{d}$ by the cows fed a diet where the RPL was estimated to supply from 15 to $21 \mathrm{~g}$ of additional intestinally absorbable Lys compared with the control diet. Because Lys was predicted to be limiting in the unsupplemented diets, it was expected that supplementing these diets with RPL would have had a positive effect on milk protein as had been observed in other studies (Blauwiekel et al., 1997; Robinson et al., 2010; Robinson et al., 2011). The combination of greater DMI and lower milk yield than anticipated resulted in diets that were predicted to have supplied sufficient amounts of Lys. Consequently, addition of RPL to the unsupplemented diets may only have resulted in an oversupply of Lys; thus, no responses were observed on milk yield or milk protein. When ingredient composition and cow responses were updated in the CPM Dairy model, supply of Lys was estimated to be 194, 170, and $156 \mathrm{~g} / \mathrm{d}$ and to meet 140,118 , and $104 \%$ of the requirement for the CON, 10DG, and 20DG diet, respectively. No effect on milk fat percentage was observed in cows fed diets supplemented with RPL, which is in agreement with others (Blauwiekel et al., 1997; Robinson et al., 2011).

\section{Plasma AA}

In lactating dairy cows, plasma concentration of the limiting AA is expected to increase only after the requirements for maintenance and production of that specific AA are met. When soybean products are replaced with increasing amounts of DDGS, plasma concentration of Lys decreases linearly and Lys is identified as the first limiting AA (Mjoun et al., 2010b). However, caution should be taken when using plasma profile of AA to determine the limiting AA, as a low plasma concentration of an AA is interpreted as having a low supply of that particular AA but this does not necessarily mean that the AA is limiting milk protein synthesis. Mjoun et al. (2010a) reported that the inclusion of DDGS in substitution of soybean products decreased the plasma concentration of Lys; however, the mammary gland adapted by increasing the extraction efficiency of Lys and both milk protein percentage and yield increased in cows fed diets with DDGS.

Differences in the diet profile of AA were reflected in the plasma profile. As expected, plasma concentration of Lys decreased (11.8\%) as DDGS inclusion increased from 0 to $20 \%$ of the diet DM, which suggests a lower supply of Lys. For other EAA, inclusion of DDGS caused the plasma concentrations of Arg, His, and Val to decrease and plasma concentrations of Leu and Met to increase. Similar to this study, Mjoun et al. (2010a) observed a decrease in the plasma concentrations of Lys and Val and an increase in the plasma concentrations of Leu and Met as DDGS was included up to $22 \%$ of the diet DM.

Factors such as fluxes from other pools and type of isomer can represent challenges to accurately interpret changes in the profile of plasma AA. Due to the small size of the plasma free-AA pool, AA fluxes from tissue pools that are larger can significantly affect the plasma concentration of AA (Bergen, 1979). The rate of disappearance of D- and L-AA can vary. For instance, the removal plasma D-Met has been shown to be 6 to 7 times slower than that of L-Met (Lapierre et al., 2012). In the present study, supplementation with RPL failed to increase the plasma concentration of Lys. Updated predictions by the CPM Dairy model showed 
that unsupplemented diets supplied enough MP-Lys to meet animal requirements; thus, providing additional MP-Lys via the RPL product should have increased the plasma concentration of Lys. One potential reason why no differences were observed could have been that the RPL product did not deliver the estimated amount of MP-Lys. It was not an objective of this study to evaluate the Lys bioavailability of the RPL product, but issues related to an inadequate rumen protection or incomplete intestinal release may have resulted in a lower bioavailability value than the one used, thus overestimating the MP-Lys supplied by the product. Another reason may have been related to the sampling regimen, as it is possible that sampling at 8 and $12 \mathrm{~h}$ after feeding was not adequate to find the time where plasma concentration of Lys peaked in the cows fed diets with RPL. For instance, Koenig and Rode (2001) observed that it was not until after 9 to $12 \mathrm{~h}$ postfeeding when plasma concentrations of Met between cows that were orally dosed with 0 or $20 \mathrm{~g}$ of rumen-protected Met differed. If samples would have only been taken before $9 \mathrm{~h}$, the authors would have concluded that the concentration of plasma Met did not differ between those treatments. A decrease in the plasma concentration of the other AA when the predicted limiting AA is supplemented has been used as an indicator that the supplemented AA was in fact limiting (Schwab et al., 1992). Assuming that the RPL product delivered the estimated MP-Lys, supplementation with RPL did not have an effect on the concentration of other AA, as updated predictions suggest that Lys was not limiting. Practically, although the inclusion of DDGS will usually result in a reduction of Lys supply, the results of this study suggest that this does not negatively affect milk protein.

\section{CONCLUSIONS}

Inclusion of DDGS up to $20 \%$ of the diet DM did not compromise intake, milk yield, or composition by the cows. Despite a decrease in plasma concentration of Lys as DDGS inclusion increased, diets with DDGS can still deliver sufficient amounts of Lys to maintain and even increase milk protein percentage and yield. Replacing feeds higher in Lys such as blood meal and soy-based products with DDGS in the concentrate portion of the diet not only decreased the plasma concentration of Lys but also affected the status of other EAA. As inclusion of DDGS increased, plasma concentrations of Arg, His, and Val decreased and Leu and Met concentrations increased. Updated predictions by the CPM Dairy model suggested that unsupplemented diets met the MP-Lys requirements of the animals; thus, supplementation with RPL was not beneficial.

\section{REFERENCES}

Anderson, J. L., D. J. Schingoethe, K. F. Kalscheur, and A. R. Hippen. 2006. Evaluation of dried and wet distillers grains included at two concentrations in the diets of lactating dairy cows. J. Dairy Sci. 89:3133-3142.

AOAC International. 2000. Official Methods of Analysis. 17th ed. AOAC International, Gaithersburg, MD.

AOAC International. 2006. Official Methods of Analysis. 18th ed. AOAC International, Gaithersburg, MD.

Bergen, W. G. 1979. Free amino acids in blood of ruminants-Physiological and nutritional regulation. J. Anim. Sci. 49:1577-1589.

Blauwiekel, R., S. Xu, J. H. Harrison, K. A. Loney, R. E. Riley, and M. C. Calhoun. 1997. Effect of whole cottonseed, gossypol, and ruminally protected lysine supplementation on milk yield and composition. J. Dairy Sci. 80:1358-1365.

Boston, R. C., D. G. Fox, C. J. Sniffen, R. Janczewski, R. Munson, and W. Chalupa. 2000. The conversion of a scientific model describing dairy cow nutrition and production to an industry tool: The CPM Dairy project. Pages 361-377 in Modelling Nutrient Utilization in Farm Animals. J. P. McNamara, J. France, and D. Beever, ed. CABI Publishing, Oxford, UK.

Christen, K. A., D. J. Schingoethe, K. F. Kalscheur, A. R. Hippen, K. K. Karges, and M. L. Gibson. 2010. Response of lactating dairy cows to high protein distillers grains or 3 other protein supplements. J. Dairy Sci. 93:2095-2104.

Fekkes, D. 1996. State-of-the-art of high-performance liquid chromatographic analysis of amino acids in physiological samples. J. Chromatogr. B Biomed. Appl. 682:3-22.

Frank, B., and C. Swensson. 2002. Relationship between content of crude protein in rations for dairy cows and milk yield, concentration of urea in milk and ammonia emissions. J. Dairy Sci. $85: 1829-1838$

Hansen, S. L., and J. W. Spears. 2009. Bioaccessibility of iron from soil is increased by silage fermentation. J. Dairy Sci. 92:2896-2905.

Heinrichs, A. J., and P. J. Kononoff. 2002. Evaluating particle size of forages and TMRs using the New Penn State Forage Particle Separator. Tech. Bull. DAS 02-42. Pennsylvania State Univ., College Agric. Sci., Cooperative Ext., University Park.

Hristov, A. N., J. K. Ropp, K. L. Grandeen, S. Abedi, R. P. Etter, A. Melgar, and A. E. Foley. 2005. Effect of carbohydrate source on ammonia utilization in lactating dairy cows. J. Anim. Sci. $83: 408-421$.

Hubbard, K. J., P. J. Kononoff, A. M. Gehman, J. M. Kelzer, K. Karges, and M. L. Gibson. 2009. The effect of feeding high-protein distillers dried grains on milk production of Holstein cows. J. Dairy Sci. 92:2911-2914.

Janicek, B. N., P. J. Kononoff, A. M. Gehman, and P. H. Doane. 2008. The effect of feeding dried distillers grains plus solubles on milk production and excretion of urinary purine derivatives. J. Dairy Sci. 91:3544-3553.

Kelzer, J. M., P. J. Kononoff, L. O. Tedeschi, T. C. Jenkins, K. Karges, and M. L. Gibson. 2010. Evaluation of protein fractionation and ruminal and intestinal digestibility of corn milling co-products. J. Dairy Sci. 93:2803-2815.

Kleinschmit, D. H., D. J. Schingoethe, K. F. Kalscheur, and A. R. Hippen. 2006. Evaluation of various sources of corn dried distillers grains plus solubles for lactating dairy cattle. J. Dairy Sci. 89:4784-4794

Koenig, K. M., and L. M. Rode. 2001. Ruminal degradability, intestinal disappearance, and plasma methionine response of rumenprotected methionine in dairy cows. J. Dairy Sci. 84:1480-1487.

Lapierre, H., G. Holtrop, A. G. Calder, J. Renaud, and G. E. Lobley. 2012. Is D-methionine bioavailable to the dairy cow? J. Dairy Sci. 95:353-362.

Mjoun, K., K. F. Kalscheur, A. R. Hippen, and D. J. Schingoethe. 2010a. Performance and amino acid utilization of early lactation dairy cows fed regular or reduced-fat dried distillers grains with solubles. J. Dairy Sci. 93:3176-3191.

Mjoun, K., K. F. Kalscheur, A. R. Hippen, D. J. Schingoethe, and D. E. Little. 2010b. Lactation performance and amino acid utilization 
of cows fed increasing amounts of reduced-fat dried distillers grains with solubles. J. Dairy Sci. 93:3176-3191.

NRC. 2001. Nutrient Requirements of Dairy Cattle. 7th rev. ed. Natl. Acad. Press, Washington, DC.

Oetzel, G. R., F. P. Villalba, W. J. Goodger, and K. V. Nordlund. 1993. A comparison of on-farm methods for estimating the drymatter content of feed ingredients. J. Dairy Sci. 76:293-299.

Owen, F. G., and L. L. Larson. 1991. Corn distillers dried drains versus soybean meal in lactation diets. J. Dairy Sci. 74:972-979.

Patton, R. A. 2010. Effect of rumen-protected methionine on feed intake, milk production, true milk protein concentration, and true milk protein yield, and the factors that influence these effects: A meta-analysis. J. Dairy Sci. 93:2105-2118.

Robinson, P. H., N. Swanepoel, and E. Evans. 2010. Effects of feeding a ruminally protected lysine product, with or without isoleucine, valine and histidine, to lactating dairy cows on their productive performance and plasma amino acid profiles. Anim. Feed Sci. Technol. 161:75-84.

Robinson, P. H., N. Swanepoel, I. Shinzato, and S. O. Juchem. 2011. Productive responses of lactating dairy cattle to supplementing high levels of ruminally protected lysine using a rumen protection technology. Anim. Feed Sci. Technol. 168:30-41.

SAS Institute. 2008. SAS/STAT ${ }^{\circledR} 9.2$ User's Guide. SAS Institute Inc., Cary, NC.

Sawada, K., K. Nagano, and N. Nishino. 2012. The relationship between plasma 3-methylhistidine concentration and forage intake in early lactating dairy cows. Livest. Sci. 143:278-282.
Schingoethe, D. J., K. F. Kalscheur, A. R. Hippen, and A. D. Garcia 2009. Invited review: The use of distillers products in dairy cattle diets. J. Dairy Sci. 92:5802-5813.

Schwab, C. G. 2010. Balancing diets for amino acids: Nutritional, environmental and financial implications. Pages 1-13 in Proc. TriState Dairy Nutr. Conf., Ft. Wayne, IN.

Schwab, C. G., C. K. Bozak, N. L. Whitehouse, and M. M. A. Mesbah. 1992. Amino acid limitation and flow to duodenum at four stages of lactation. 1. Sequence of lysine and methionine limitation. J. Dairy Sci. 75:3486-3502.

Swanepoel, N., P. H. Robinson, and L. J. Erasmus. 2010. Amino acid needs of lactating dairy cows: Impact of feeding lysine in a ruminally protected form on productivity of lactating dairy cows. Anim. Feed Sci. Technol. 157:79-94.

Van Soest, P. J., J. B. Robertson, and B. A. Lewis. 1991. Methods for dietary fiber, neutral detergent fiber, and nonstarch polysaccharides in relation to animal nutrition. J. Dairy Sci. 74:3583-3597.

Wildman, E. E., G. M. Jones, P. E. Wagner, R. L. Boman, H. F Troutt Jr., and T. N. Lesch. 1982. A dairy cow body condition scoring system and its relationship to selected production characteristics. J. Dairy Sci. 65:495-501.

Zhang, S. Z., G. B. Penner, W. Z. Yang, and M. Oba. 2010. Effects of partially replacing barley silage or barley grain with dried distillers grains with solubles on rumen fermentation and milk production of lactating dairy cows. J. Dairy Sci. 93:3231-3242. 